\title{
Deconvolution Under Normalized Autocorrelation Constraints
}

\author{
B. Baygün, F. J. Kuchuk, and O. Arikan
}

\begin{abstract}
In this paper we describe a time domain algorithm for determining the influence function from the measured input and output signals of the system. The deconvolution, which is a highly unstable inverse problem with measurement errors, is an important step for obtaining the system's influence function that provides insight about flow regimes normally masked by the time-dependent input signal. The algorithms presented for deconvolution in the literature are generally based on data reduction, with the exception of constrained deconvolution methods.

We propose a constrained least-squares deconvolution method to reconstruct the influence function from noisy data. The constraints are the lower bounds on the first few lags of the normalized autocorrelation coefficients of the influence function. The lower bounds may represent known or desirable smoothness properties of the function. By choosing the constraint values larger, a smoother deconvolution can be obtained. We also impose an energy constraint on the derivative of the reconstructed signal for further regularization.
\end{abstract}

\section{Introduction}

Deconvolution is a signal processing method in which the effect of the time-dependent input signal is extracted from the output signal. In testing, it is defined as determining the influence function or unit response behavior of a system at the wellbore (the constant rate behavior) from measured downhole pressure and flow rate. ${ }^{1}$ For convenience, this deconvolution is called pressurerate deconvolution. Recently, Goode etal. $^{2}$ presented a different approach using pressure signals from the horizontal and vertical probes of the multiprobe wireline formation tester ${ }^{3}$ to obtain the ratio impulse function of the system. The deconvolution method described by Goode $e t a l .{ }^{2}$ is called pressure-pressure deconvolution in this paper. The pressure-pressure deconvolution as shown in Appendix A can easily be extended to other well-testing problems, such as interference testing between wells. For both methods, the deconvolution operation can be defined as obtaining solutions for convolution type linear Volterra integral equations that can be written as

$$
p(t)=\int_{0}^{t} f(\tau) \mathcal{G}(t-\tau) d \tau
$$

Copyright 1997, Society of Petroleum Engineers, Inc.

Paper first presented at the SPE Annual Technical Conference \& Exhibition held in New Orieans, U.S.A., 25-28 September 1995.

Received for review, August 19, 1996

Accepted for publication, January 23, 1997

Camera-ready copy received, March 31, 1997

SPE 28405
For convenience, we use $p$ instead of $\Delta p$. The kernel $\mathcal{G}$ of the convolution integral is called the impulse response or the ratio of the impulse responses evaluated at two different locations (see Appendix A). Whether the kernel is an impulse response or a ratio of impulse responses, the impulse response is a solution of the diffusivity equation for a Neumann-type internal boundary condition. As discussed by Kuchuk et al. ${ }^{1}$ the deconvolution method given in this paper can also be applied to Dirichlet-type problems. The quantities $p$ and $f$ in Eq. 1 are measured as functions of time.

The deconvolved kernel $\mathcal{G}$, and its integral (unit response) and logarithmic derivative forms $(t \times \mathcal{G})$ are used in system identification, which is the first step of the well test interpretation. Many different deconvolution algorithms can be developed using different operational techniques. The most appropriate method for a particular testing problem depends on the behavior and the noise characteristics of the measurements. In the absence of noise, numerical algorithms for approximating the solution of Eq. 1 for the kernel $\mathcal{G}$ would be relatively trivial. However, as discussed in Kuchuk et al., ${ }^{1}$ even small amounts of noise in the flow rate have a detrimental effect on the solution. Consequently, Coats etal. ${ }^{4}$ used a linear programming method with a number of constraints in order to smooth the solution. An alternative constrained deconvolution method using linear least-square minimization was presented by Kuchuk et al. ${ }^{1}$ The latter method gives a smooth solution for the integral of $\mathcal{G}$, but $\mathcal{G}$ itself oscillates mildly. The oscillatory nature of the solution from the method of Kuchuk etal. ${ }^{1}$ sometimes hinders the identification step. 
In this paper, we propose a novel constrained leastsquares deconvolution method to reconstruct the influence function from noisy data. The constraints are the lower bounds on the first few lags of the normalized autocorrelation coefficients of the influence function. The lower bounds may represent known or desirable smoothness properties of the function. The inclusion of the autocorrelation constraints generates a regularization effect on the deconvolution. Numerical results indicate that the proposed approach can significantly improve the quality of the deconvolution.

\section{Formulation of the Problem}

We first consider a discretized version of Eq. 1. We assume that the quantities $p(t)$ and $f(t)$ are measured at times $t_{0}, t_{1}, \ldots, t_{N}$. It is desirable to find the values of $\mathcal{G}$ at the same time points.

An equivalent way of writing the convolution integral in Eq. 1 is

$$
p(t)=\int_{0}^{t} f(t-\tau) \mathcal{G}(\tau) d \tau
$$

A discrete trapezoid approximation to the integral in Eq. 2 would be

$$
p\left(t_{n}\right)=\sum_{k=0}^{n-1} f\left(t_{n}-t_{k}\right) \mathcal{G}\left(t_{k}\right) \Delta(k), 1 \leq n \leq N
$$

where $\Delta(k)$ is a scaling factor that is a function of the sampling interval lengths $t_{k}-t_{k-1}$ and $t_{k+1}-t_{k}\left(t_{-1} \equiv\right.$ 0 ). If the sampling times are uniformly spaced, i.e., the length of the sampling interval $t_{k}-t_{k-1}$ is constant over $k$, then the values $f\left(t_{n}-t_{k}\right)$ correspond to the measured values of $f(t)$, only in reverse order. On the other hand, if the sampling times are not uniformly spaced, then the time value $\left(t_{n}-t_{k}\right)$ does not necessarily correspond to one of the original sampling times $t_{0}, \ldots, t_{N}$. In the latter case, the values $f\left(t_{n}-t_{k}\right)$ should be approximated from the measured values $f\left(t_{0}\right), \ldots, f\left(t_{N}\right)$. In our implementation, we compute the values $f\left(t_{n}-t_{k}\right)$ using splines.

Eq. 3 can be written using matrix notation as

$$
\mathbf{y}=\mathbf{H} \mathbf{x}+\mathbf{w}
$$

where $\mathbf{y}$ is the observed output signal (pressure measurements) vector $\left[p\left(t_{1}\right), \ldots, p\left(t_{N}\right)\right]^{T}$ (the superscript $T$ denotes matrix transpose), $\mathbf{H}$ is a lower-triangular matrix representing the time variation of $f(t)$ (the $n^{\text {th }}$ row of $\mathbf{H}$ is $\left.\left[f\left(t_{n}-t_{0}\right) \times \Delta(0), \ldots, f\left(t_{n}-t_{n-1}\right) \times \Delta(n-1)\right]\right)$, $\mathbf{x}$ is the signal vector $\left[\mathcal{G}\left(t_{0}\right), \ldots, \mathcal{G}\left(t_{N-1}\right)\right]^{T}$ to be reconstructed (the desired deconvolution result), and $w$
$(N \times 1)$ is the observation noise vector. We use boldface lower and upper case characters to denote vectorial and matrix variables, respectively. $w$ represents any discrepancy between the convolutional model and the actual observation $\mathbf{y}$. We seek the vector $\mathbf{x}$ that best matches the observed $\mathbf{y}$ in the least-squares sense, i.e.,

$$
\min _{\mathbf{x}}\left\{\left.\|\mathbf{y}-\mathbf{H} \mathbf{x}\|\right|^{2}\right\} \text {. }
$$

When there is no noise ( $w \equiv 0$ ), the solution of the minimization problem in Eq. 5 can be found by premultiplying $\mathbf{y}$ by the inverse of the matrix $\mathbf{H}$. Because $\mathbf{H}$ is lower-triangular, the inverse exists unless some of the diagonal elements of $\mathbf{H}$ are zero. However, if the condition number (the ratio of the largest singular value of $\mathbf{H}$ to the smallest singular value) of $\mathbf{H}$ is high, the matrix inversion could be highly unstable, giving rise to an oscillatory deconvolution result. An alternative, but equivalent, recursive solution for $\mathbf{x}$ can be formulated exploiting the lower-triangular structure of $\mathbf{H}$. In this solution, the elements of $\mathbf{x}$ are obtained starting with the first row and solving for $x_{1}$, then proceeding to the second row and solving for $x_{2}$, this time using the previously computed value of $x_{1}$, and then continuing down the rows of $\mathbf{y}$ and $\mathbf{H}$. Unfortunately, this recursive solution fails with even the slightest noise on the right-hand side of Eq. 4, because the noise propagates over increasing indices of $x_{n} .{ }^{1}$ Hence, in general, this recursive solution is not preferred.

To counter the adverse effects experienced in linear inversion, some sort of regularization is usually applied. Regularization can be affected by solving the minimization problem in Eq. 5 under a set of constraints. The deconvolution method we propose falls into this category. Basically, we suppress the effects of noise by imposing smoothness constraints and the effects of an illconditioned $\mathbf{H}$ matrix by imposing an energy constraint.

Before we give a precise statement of the constraints, it is worthwhile to explain the reasoning behind them. By smoothness of the signal $x_{1}, \ldots, x_{N}$ represented by the vector $\mathrm{x}$, we mean that the signal does not change rapidly. In other words, for any $n, 1 \leq n \leq N-1$, we do not expect $x(n)$ to be too different from $x(n+1)$. Hence, if we compare the two vectors $\mathrm{z}_{1} \stackrel{\text { def }}{=}\left[x_{2}, x_{3}, \ldots, x_{N}\right]^{T}$ and $\mathbf{z}_{2} \stackrel{\text { def }}{=}\left[x_{1}, x_{2}, \ldots, x_{N-1}\right]^{T}$, they should be somewhat similar. The maximum similarity would be attained if $\mathbf{z}_{1}=\mathbf{z}_{2}$, in which case $x_{1}=x_{2}=\ldots=x_{N}$, i.e., if we have a constant signal. The minimum similarity would be attained if $\mathbf{z}_{1}=-\mathbf{z}_{2}$, in which case $x_{1}=-x_{2}=x_{3}=\ldots=(-1)^{(N-1)} x_{N}$. Clearly, in the latter case unless $\mathbf{x} \equiv \mathbf{0}$, we have a highly oscillatory signal, which is undesirable. The two extreme cases suggest a mechanism to control the level of smoothness in $\mathbf{x}$. First observe that due to the Cauchy-Schwarz 
inequality, ${ }^{5}$ the following inequality always holds:

$$
\left|\frac{\sum_{n=1}^{N-1} x(n) x(n+1)}{\left(\sum_{n=1}^{N-1}(x(n))^{2}\right)^{1 / 2}\left(\sum_{n=2}^{N}(x(n))^{2}\right)^{1 / 2}}\right| \leq 1
$$

Therefore, we can control the smoothness by imposing the following constraint:

$$
\frac{\sum_{n=1}^{N-1} x(n) x(n+1)}{\left(\sum_{n=1}^{N-1}(x(n))^{2}\right)^{1 / 2}\left(\sum_{n=2}^{N}(x(n))^{2}\right)^{1 / 2}} \geq \theta_{1}
$$

and varying the value of $\theta_{1}$ between -1 and 1 . The higher the value of $\theta_{1}$, the smoother the vector $\mathbf{x}$.

Having made this observation, we can generalize the smoothness constraints from adjacent samples $x(n)$ and $x(n+1)$ to samples that are sufficiently close, i.e. $x(n)$ and $x(n+m)$, for $1 \leq m \leq M_{1}$ and $1 \leq n \leq N-m$. More specifically, to impose similarity on samples that are $m$ points apart, we can use the following constraint:

$$
\begin{gathered}
\sum_{n=1}^{N-m} x(n) x(n+m) \\
\left(\sum_{n=1}^{N-m}(x(n))^{2}\right)^{1 / 2}\left(\sum_{n=m+1}^{N}(x(n))^{2}\right)^{1 / 2} \geq \theta_{m}
\end{gathered}
$$

where $\theta_{m} \leq 1$. Similarly to Eq. 6 , one knows from the Cauchy-Schwarz inequality that the fraction in Eq. 8 is upper-bounded by 1 . Therefore, higher values of $\theta_{m}$ give rise to greater similarity between samples that are $m$ points apart.

In our applications, we found that for the purpose of obtaining the logarithmic derivative $t \times \mathcal{G}$ (wehere $\mathcal{G}$ is usually the ratio of two impulse responses and $t$ is time), it is better to impose smoothness constraints of the form given in Eq. 8 not on $\mathcal{G}$ (i.e. on $\mathbf{x}$ ) but on $t \times \mathcal{G}$. Furthermore, we made a slight modification on the denominator. More specifically, we used the following set of constraints:

$$
\frac{\sum_{n=1}^{N-m} x_{t}(n) x_{t}(n+m)}{\sum_{n=1}^{N}\left(x_{t}(n)\right)^{2}} \geq \theta_{m}, \quad 1 \leq m \leq M_{1}
$$

where $\mathbf{x}_{t} \stackrel{\text { def }}{=}[t(1) \times x(1), \ldots, t(N) \times x(N)]^{T}$. In analogy with the original development of a similar set of smoothness constraints in the context of stochastic signal reconstruction in Arlkan and Baygün, ${ }^{6}$ we refer to the constraints in Eq. 9 as "autocorrelation constraints."

The constraints in Eq. 9 impose smoothness on the solution. To further reduce the problems associated with the inversion of an ill-conditioned matrix $\mathbf{H}$, we use an energy constraint on the derivative $\mathbf{d}_{x}$ of $\mathbf{x}$ :

$$
\sum_{n=1}^{N}\left(d_{x}(n)\right)^{2} \leq \alpha
$$

where $\mathrm{d}_{x} \stackrel{\text { def }}{=}\left[x_{t}(2)-x_{t}(1), \ldots, x_{t}(N)-x_{t}(N-1)\right]^{T}$.

The formal statement of the proposed constrained deconvolution method, which combines the constraints in Eqs. 9 and 10, is as follows:

$$
\begin{aligned}
\begin{aligned}
\operatorname{minimize} \\
\text { subject to: }
\end{aligned} & \left\{\|\mathbf{y}-\mathbf{H} \mathbf{x}\|^{2}\right\} \\
\text { i. } & \frac{\sum_{n=1}^{N-m} x(n) x(n+m)}{\sum_{n=1}^{N}(x(n))^{2}} \geq \theta_{m}, \\
& 1 \leq m \leq M_{1} \\
\text { ii. } \quad & \sum_{n=1}^{N}\left(d_{x}(n)\right)^{2} \leq \alpha .
\end{aligned}
$$

\section{Solution of the Constrained Least Squares Problem}

The constrained least-squares problem in Eqs. 11 through 13 can be reformulated using the method of Lagrange multipliers. It can be shown that the cost function in Eq. 11 and the constraints in Eqs. 12 and 13 satisfy the required regularity assumptions so that the first order necessary conditions on the following Lagrangian function characterize the constrained least-squares problem.

$$
\mathcal{L}(\mathbf{x}, \boldsymbol{\lambda})=\|\mathbf{y}-\mathbf{H} \mathbf{x}\|^{2}+\sum_{m=1}^{M} \lambda_{m} c_{m}(\mathbf{x})
$$

where $M=M_{1}+1, \lambda \stackrel{\text { def }}{=}\left[\lambda_{1}, \ldots, \lambda_{M}\right]^{T}$ is the vector of Lagrange multipliers, $c_{1}, \ldots, c_{M}$ are the smoothness constraints on $\mathbf{x}$, and $c_{M}$ is the energy constraint:

$$
c_{m}(\mathbf{x})=\left\{\begin{array}{r}
\theta_{m} \sum_{n=1}^{N}(t(n) x(n))^{2} \\
-\sum_{n=1}^{N=m} t(n) x(n) x(n+m) t(n+m), \\
1 \leq m \leq M-1 \\
\sum_{n=1}^{N}(t(n+1) x(n+1)-t(n) x(n))^{2}-\alpha, \\
m=M .
\end{array}\right.
$$

The first order necessary conditions (Kuhn-Tucker conditions) for optimality are as follows.

$$
\begin{aligned}
\nabla_{\mathbf{x}} \mathcal{L}(\mathbf{x}, \boldsymbol{\lambda})=\mathbf{0} & \\
\lambda_{m} \geq 0, & 1 \leq m \leq M \\
c_{m}(\mathbf{x}) \geq 0, & 1 \leq m \leq M \\
\lambda_{m} c_{m}(\mathbf{x})=0, & 1 \leq m \leq M
\end{aligned}
$$

The first of these conditions provides a solution for $\mathbf{x}$ in terms of $\boldsymbol{\lambda}$. We show in Appendix B that this solution has the form

$$
\mathbf{x}(\boldsymbol{\lambda})=\left(\mathbf{H}^{T} \mathbf{H}+\boldsymbol{\Lambda}\right)^{-1} \mathbf{H}^{T} \mathbf{y}
$$


where $\boldsymbol{\Lambda}$ is a matrix with entries that are functions of $\lambda$.

We can now write the dual of the original constrained least-squares problem in Eqs. 11 through 13:

$$
\begin{aligned}
& \operatorname{minimize} \boldsymbol{\lambda}\|\mathbf{y}-\mathbf{H} \mathbf{x}(\boldsymbol{\lambda})\|^{2} \\
& \text { subject to: } \\
& \lambda_{m} \geq 0, \quad 1 \leq m \leq M \\
& c_{m}(\mathbf{x}(\boldsymbol{\lambda})) \geq 0, \quad 1 \leq m \leq M \\
& \lambda_{m} c_{m}(\mathbf{x}(\boldsymbol{\lambda}))=0, \quad 1 \leq m \leq M .
\end{aligned}
$$

The dual problem has an important computational advantage over the original problem. While the original problem is an optimization over $N$ variables $x_{1}, \ldots, x_{N}$, the dual problem is an optimization over $M$ variables $\lambda_{1}, \ldots, \lambda_{M}$, where typically $M \ll N$. For instance, in our numerical examples $N$ is between 150 and 200, while $M$ is 1 or 2 .

\section{Results and Discussion}

In this section, we apply our method to a few examples to demonstrate the improvement that can be achieved by using the constraints in the deconvolution. Let us suppose that we conduct a flow test with a dual-packer module of the multiprobe wireline formation tester (see Zimmerman et al..$^{3}$ for more details about the tool) in a homogeneous formation. For this example, the input flow rate and the output packer interval pressure measurements are shown in Fig. 1. In addition to these measurements, let us assume that the pressure is also measured at $5 \mathrm{ft}$ above from the center of the packer module with an observation probe. Figure 1 also presents the observation probe measurements. The distance between the packers (the length of the open interval) is $3.2 \mathrm{ft}$ with a well radius of $0.35 \mathrm{ft}$. The formation and fluid properties for the example are $k_{h}=100 \mathrm{md}, k_{v}=10 \mathrm{md}$, $h=10 \mathrm{ft}, z_{w}=5 \mathrm{ft}$ (the vertical distance from the center of the open interval to the bottom of the formation), $\phi=0.20, \mu=1 \mathrm{cp}$, and $c_{t}=1.0 \times 10^{-5} \mathrm{psi}^{-1}$.

As described in Appendix A, let us obtain the $\mathcal{G}$ function for this formation from the pressure-pressure deconvolution of the pressure measurements at both packer and observation probes. To achieve acceptable computation speed, we limit the number of constraints to $M \leq 2$. In other words, we consider a smoothness constraint only on consecutive samples and/or the derivative energy constraint. We present both noiseless and noisy examples. The data are synthetic data and the noise is assumed to be uniformly distributed within a specified band about the true convolution result $\mathbf{H x}$. The result of the deconvolution is denoted by $\hat{\mathbf{x}}$.

We start with the noiseless case to emphasize that although the inverse of the $\mathbf{H}$ matrix exists theoretically, there may be computational problems in practice.

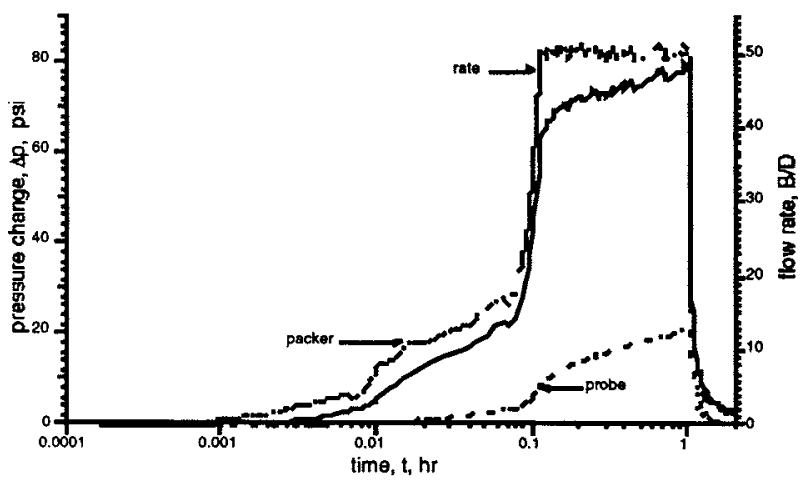

Fig. 1-Flow rate and packer and probe pressures.

The result of the direct inversion, which was extremely poor, is not shown here. In Fig. 2, we present the deconvolution result under the derivative energy constraint. As can be seen, the deconvolution result $\hat{\mathbf{x}}$ matches the true $\mathbf{x}$ very closely. We have also obtained very similar results with only the autocorrelation constraint.

We noted previously that the recursive computation method gives a reliable deconvolution result in the noiseless case. However, because the recursive method suffers heavily with the addition of even a little noise, that method will not be considered in our examples.

Next, we look at the noisy case. The result for $1 \%$ additive noise using the autocorrelation constraint only is given in Fig. 3. Note that the general trend of $\mathbf{x}$ is captured in the deconvolution result $\hat{\mathbf{x}}$. The oscillations towards the end can be suppressed by adding the energy constraint on the derivative. Fig. 3 also shows the result with both derivative energy and autocorrelation constraints. The late-time oscillations in $\hat{\mathbf{x}}$ are now lower than before and the deconvolution result matches the original signal $\mathbf{x}$ more closely.

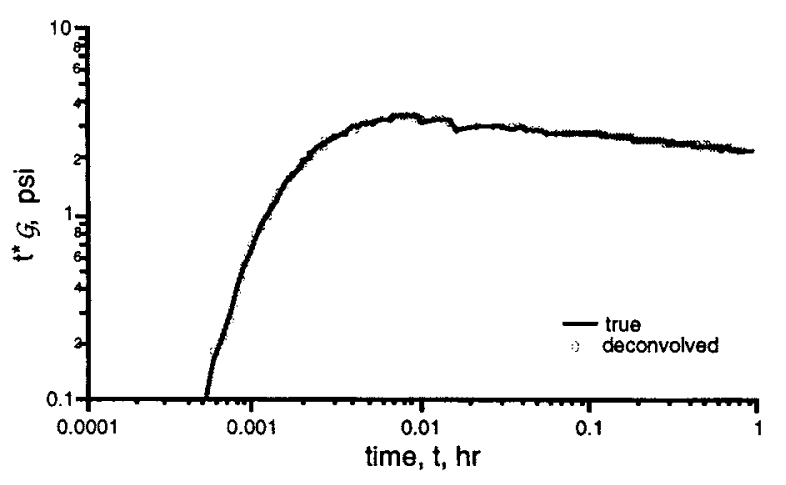

Fig. 2-True and deconvolved $t \times \mathcal{G}$ under the derivative energy constraint in the noiseless case. 


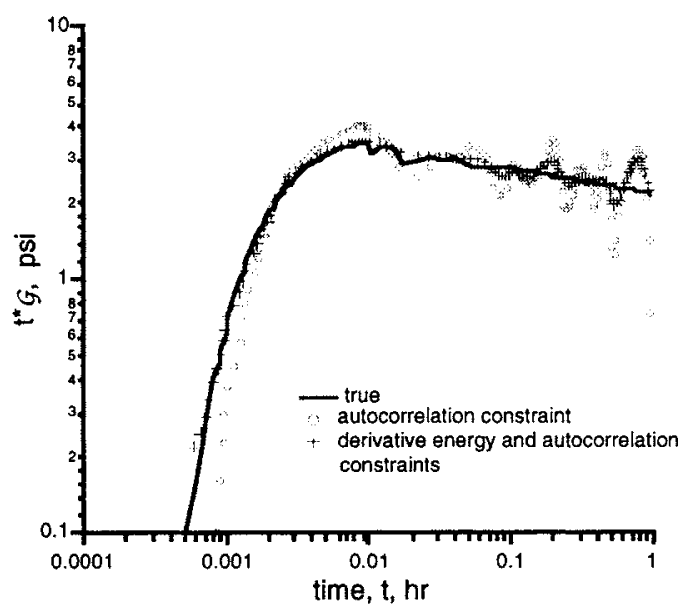

Fig. 3-True and deconvolved $t \times \mathcal{G}$ using the autocorrelation constraint and both derivative energy and autocorrelation constraints with $1 \%$ additive noise.

Figs. 4 shows the deconvolution results for $2 \%$ additive noise under each of the constraints separately as well as under both constraints. Fig. 4 shows that using only the autocorrelation constraints we get a good fit at early-time, while at late-time, we have oscillations. The important point is that the solution oscillates about the true $\mathbf{x}$. In other words, the solution does not have a bias. If we use the derivative energy constraint instead of the autocorrelation constraint, then, as Fig. 4 shows, the late-time oscillations become lower but at the expense of a mismatch at early-time. The deconvolution result can be improved by using the two constraints together, which gives us the solution depicted in Fig. 4. Simultaneous use of the two constraints provides a good fit at early-time and ensures a low level of oscillation at late-time.

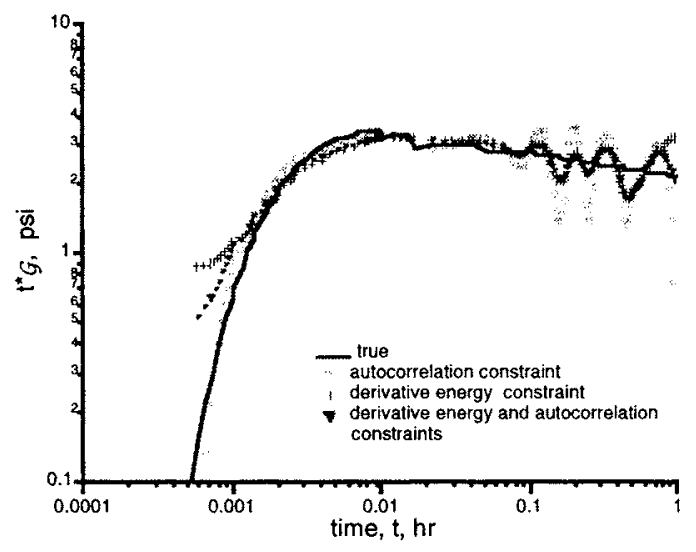

Fig. 4-True and deconvolved $t \times G$ using the autocorrelation constraint and both derivative energy and autocorrelation constraints with $2 \%$ additive noise.
In Fig. 5 we provide the results for a high noise case, where $5 \%$ noise was added to the convolution result and both energy and smoothness constraints were used. Even with significant noise, the deconvolution result shown here follows the true $\mathbf{x}$ reasonably closely. The conclusion from this and previous examples is that when both autocorrelation and energy constraints are used, the deconvolution is quite smooth and approximates the true $\mathbf{x}$ closely, even with significant measurement errors.

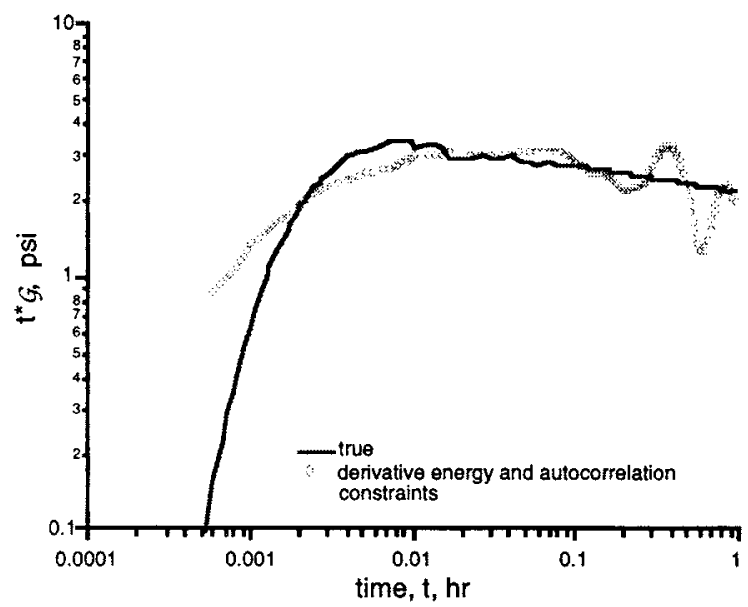

Fig. 5-True and deconvolved $t \times G$ using both derivative energy and autocorrelation constraints with $5 \%$ additive noise.

\section{Conclusions}

In this paper we present a novel time-domain algorithm for extracting the influence function from the measured input and output signals of a system. We pose the problem in a least-squares minimization framework. The novel aspect of our approach is a set of constraints that provides a regularization to the ordinary least squares solution. We consider two different constraints: autocorrelation constraints and derivative energy constraints. We present numerical examples which demonstrate that, at late-time, our deconvolution result oscillates about the true influence function, i.e., our result does not have a bias away from the true solution. Furthermore, the magnitude of the late-time oscillations, which can be high in deconvolution results obtained by other methods, are suppressed significantly by our method. Therefore, the deconvolution result describes the true influence function very closely.

\section{Nomenclature}

$\mathbf{A}=$ defined by Equation B-2

$c=$ compressibility, $\mathrm{psi}^{-1}\left[\mathrm{~Pa}^{-1}\right]$ or constraint 


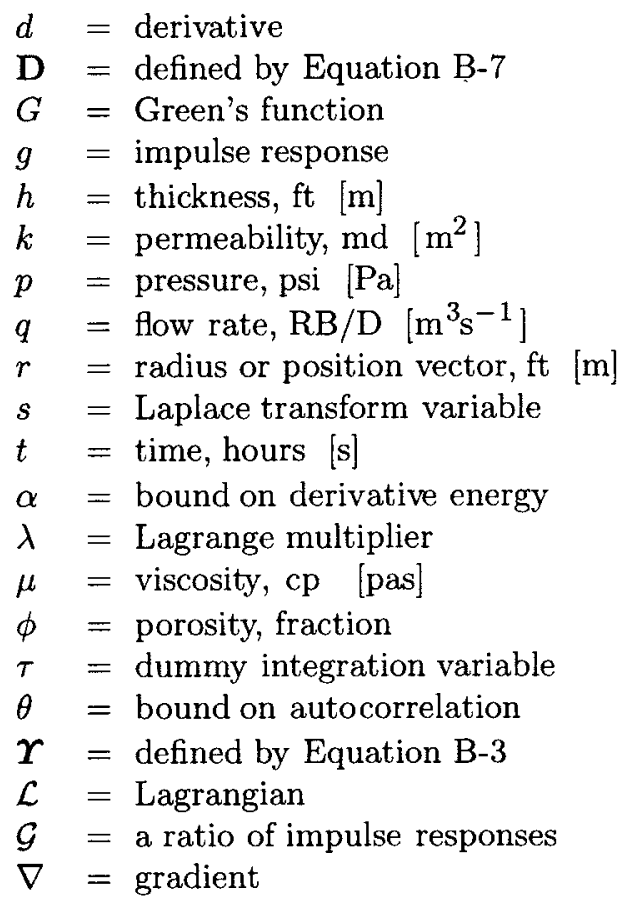

Subscripts

$$
\begin{aligned}
& h=\text { horizontal } \\
& m=\text { measured or } m-t h \\
& o=\text { initial or original } \\
& t=\text { total } \\
& v=\text { vertical } \\
& w=\text { well or wellbore } \\
& x=x \text { with respect to } \\
& \mathbf{x}=\text { with respect to } \mathbf{x}
\end{aligned}
$$

Superscripts

$$
\begin{aligned}
T & =\text { transpose } \\
& =\text { estimate (deconvolution result) }
\end{aligned}
$$

\section{Acknowledgments}

We are grateful to Schlumberger for permission to publish this paper.

\section{References}

1. Kuchuk, F. J., Carter, R. G. and Ayestaran, L.: "Deconvolution of Wellbore Pressure and Flow Rate," SPEFE (1990) 53-59.

2. Goode, P. A., Pop, J. J., and Murphy III, W. F.: "Multiple Probe Formation Testing and Vertical Reservoir Continuity," SPE 22738 presented at the SPE Annual Technical Conference and Exhibition, Dallas (Oct. 1991).

3. Zimmerman, T., MacInnis, J., Hoppe, J., Pop, J. and Long, T.: "Application of emerging wireline formation tester technologies," OSEA-90081, Eighth Offshore Southeast Conf. (Dec.), Singapore (1990).

4. Coats, K. H., Rapoport, L. A., McCord, J. R., and Drews, W. P : "Determination of Aquifer Influ-

ence Functions From Field Data," JPT (Dec. 1964) 1417-24.

5. Luenberger, D. G: Optimization by Vector Space Methods, Wiley, New York (1969).

6. Arıkan, O. and Baygün, B.: "Least-Squares Signal Reconstruction Under Normalized Autocorrelation Constraints," Proceedings of ICASSP-94, Adelaide, Australia (April 1994).

\section{Appendix A: Pressure-Pressure Deconvoptlution}

Let us consider pressure diffusion in a three-dimensional infinite (unbounded) porous medium for a flow of a slightly compressible single-phase fluid with pressure and time-invariant fluid and formation properties. The pressure distribution in a such system due to a prescribed fluid flux (a Neumann-type boundary condition which is the flow rate for our problem) at an internal boundary surface can be written as

$$
p(t, \mathbf{r})=p_{o}-\int_{\tau=0^{-}}^{t} d \tau q_{m}(\tau) g(t-\tau, \mathbf{r}), \quad(\mathrm{A}-1)
$$

where $p_{o}$ is the initial pressure, $\mathbf{r}$ is the spatial position vector, and $t$ is the time. In Eq. A-1, the flow rate (prescribed fluid flux) $q_{m}$ and the impulse response $g$ are zero for $t<0$. The expression given by Eq. A-1 is known as Duhamel's theorem. The impulse response $g$ is a solution of the diffusivity equation for a Neumann-type internal boundary condition. The pressure distribution given by Eq. A-1 can easily be generalized for multiple internal boundary surfaces in unbounded or bounded systems.

Clearly, the pressure-rate deconvolution is nothing more than obtaining $g$ in Eq. A-1 from the measured $p$ at a given $\mathbf{r}$ (at any point in the spatial domain) and $q_{m}$ measured at the internal boundary. Let us write the Laplace transform of Eq. A-1 at two different spatial locations $\mathbf{r}_{1}$ and $\mathbf{r}_{2}$ as

$$
\Delta \bar{p}\left(s, \mathbf{r}_{1}\right)=\bar{q}_{m}(s) \bar{g}\left(s, \mathbf{r}_{1}\right)
$$

and

$$
\Delta \bar{p}\left(s, \mathbf{r}_{2}\right)=\bar{q}_{m}(s) \bar{g}\left(s, \mathbf{r}_{2}\right), \quad(\mathrm{A}-3)
$$

where $\Delta p=p_{o}-p(t, \mathbf{r})$. As described by Goode et al.,$^{2}$ solving Eq. A-2 for $\bar{q}_{m}$ and substituting it in Eq. A-3, we obtain the Laplace transform of the pressure at $\mathbf{r}_{2}$ as

$$
\Delta \bar{p}\left(s, \mathbf{r}_{2}\right)=\Delta \bar{p}\left(s, \mathbf{r}_{1}\right) \overline{\mathcal{G}}(s), \quad(\mathrm{A}-4)
$$

where $\overline{\mathcal{G}}(s)=\frac{\bar{g}\left(s, \mathbf{r}_{2}\right)}{\bar{g}\left(s, \mathbf{r}_{1}\right)}$ that is called $\mathcal{G}$ function. ${ }^{2}$ In the time domain, Eq. A-4 can be written as

$$
\Delta p\left(t, \mathbf{r}_{2}\right)=\int_{\tau=0}^{t} d \tau \Delta p\left(\tau, \mathbf{r}_{1}\right) \mathcal{G}(t-\tau) . \quad(\mathrm{A}-5)
$$


Obtaining $\mathcal{G}$ in Eq. A-5 from measured $\Delta p\left(t, \mathbf{r}_{1}\right)$ and $\Delta p\left(t, \mathbf{r}_{2}\right)$ is called pressure-pressure deconvolution.

For an interference test, $\Delta p\left(t, \mathbf{r}_{1}\right)$ can be the pressure at the pulse well, while $\Delta p\left(t, \mathrm{r}_{2}\right)$ can be the pressure at the observation well. For a vertical interference test within the wellbore, $\Delta p\left(t, \mathbf{r}_{1}\right)$ can be the pressure at the pulse (injection or production) interval while $\Delta p\left(t, \mathbf{r}_{2}\right)$ can be the pressure at the observation interval. For this test configuration, the pulse interval is normally isolated from the observation interval by a packer. For the multiprobe wireline formation tester, $\Delta p\left(t, \mathbf{r}_{1}\right)$ can be the pressure at the sink probe or the horizontal probe, while $\Delta p\left(t, \mathbf{r}_{2}\right)$ can be the pressure at the observation probe.

\section{Appendix B: Solution of the First Kuhn-Tucker Equation}

The smoothness constraints $c_{m}(\mathbf{x}), m=1, \ldots, M-1$ in Eq. 15 can be written as

$$
c_{m}(\mathbf{x})=\theta_{m} \mathbf{x}^{T}\left(\boldsymbol{r}^{T} \boldsymbol{Y}\right) \mathbf{x}-\mathbf{x}^{T} \boldsymbol{\Upsilon}^{T} \mathbf{A}_{m} \boldsymbol{\Upsilon} \mathbf{x}, \quad(\mathrm{B}-1)
$$

where

$$
\begin{gathered}
\mathbf{A}_{m} \stackrel{\text { def }}{=}\left[\begin{array}{cc}
\mathbf{0} & \mathcal{I}_{N-m} \\
\mathbf{0} & \mathbf{0}
\end{array}\right], \\
\boldsymbol{\Upsilon} \stackrel{\text { def }}{=}\left[\begin{array}{cccc}
t(1) & 0 & \ldots & 0 \\
\vdots & \vdots & \ddots & \vdots \\
0 & 0 & \ldots & t(N)
\end{array}\right]
\end{gathered}
$$

and $\mathcal{I}_{N-m}$ is the identity matrix of order $(N-m)$. Now note that for any $N$-vector $\mathbf{z}$ the following identity holds.

$$
\mathbf{z}^{T} \mathbf{A}_{m} \mathbf{z}=\frac{1}{2} \mathbf{z}^{T}\left(\mathbf{A}_{m}+\mathbf{A}_{m}^{T}\right) \mathbf{z} .
$$

Therefore, taking $\mathbf{z}=\boldsymbol{\Upsilon} \mathbf{x}$ for $1 \leq m \leq M-1$ we obtain

$$
\begin{aligned}
c_{m}(\mathbf{x}) & =\theta_{m} \mathbf{x}^{T}\left(\boldsymbol{r}^{T} \boldsymbol{\Upsilon}\right) \mathbf{x}-\frac{1}{2} \mathbf{x}^{T} \boldsymbol{r}^{T}\left(\mathbf{A}_{m}\right. \\
& \left.+\mathbf{A}_{m}^{T}\right) \boldsymbol{r} \mathbf{x}=\mathbf{x}^{T} \boldsymbol{r}^{T}\left[\theta_{m} \mathcal{I}_{N}-\frac{1}{2}\left(\mathbf{A}_{m}\right.\right. \\
& \left.\left.+\mathbf{A}_{m}^{T}\right)\right] \boldsymbol{r} \mathbf{x} .
\end{aligned}
$$

Similarly, the final constraint $c_{M}(\mathbf{x})=\mathbf{d}_{x}^{T} \mathbf{d}_{x}-\alpha$ can be written a

$$
c_{M}(\mathbf{x})=\mathbf{x}^{T}\left(\boldsymbol{r}^{T} \mathbf{D}^{T} \mathbf{D} \boldsymbol{\Upsilon}\right) \mathbf{x}-\alpha
$$

where

$$
\mathbf{D} \stackrel{\text { def }}{=}\left[\begin{array}{ccccc}
-1 & 1 & 0 & \ldots & 0 \\
0 & -1 & 1 & 0 & \ldots \\
\vdots & \vdots & \vdots & \ddots & \ldots \\
0 & \ldots & 0 & -1 & 1
\end{array}\right] . \quad(B-7)
$$

is the $(N-1) \times N$ derivative matrix. Using Eqs. B-5 and B-6 we can rewrite the Lagrangian function $\mathcal{L}(\mathbf{x}, \lambda)$ defined by Eq. 14 as follows:

$$
\begin{aligned}
& \mathcal{L}(\mathbf{x}, \boldsymbol{\lambda})=\|\mathbf{y}-\mathbf{H} \mathbf{x}\|^{2}+\mathbf{x}^{T} \boldsymbol{r}^{T}\left[\mathcal{I}_{N}\left(\sum_{m=1}^{M-1} \lambda_{m} \theta_{m}\right)\right. \\
& \left.+\lambda_{M} \mathbf{D}^{T} \mathbf{D}-\frac{1}{2} \sum_{m=1}^{M-1} \lambda_{m}\left(\mathbf{A}_{m}+\mathbf{A}_{m}^{T}\right)\right] \boldsymbol{r} \mathbf{x}-\lambda_{M} \alpha .
\end{aligned}
$$

Now let

$$
\begin{aligned}
\boldsymbol{\Lambda}^{\mathrm{def}} & =\mathcal{I}_{N}\left(\sum_{m=1}^{M-1} \lambda_{m} \theta_{m}\right)+\lambda_{M} \mathbf{D}^{T} \mathbf{D} \\
& -\frac{1}{2} \sum_{m=1}^{M-1} \lambda_{m}\left(\mathbf{A}_{m}+\mathbf{A}_{m}^{T}\right) .
\end{aligned}
$$

By using the $\boldsymbol{A}$ matrix, we can express the Lagrangian function in Eq. B-8 as

$$
\mathcal{L}(\mathbf{x}, \lambda)=\|\mathbf{y}-\mathbf{H} \mathbf{x}\|^{2}+\mathbf{x}^{T} \boldsymbol{\Lambda} \mathbf{x}-\lambda_{M} \alpha . \quad(\mathrm{B}-10)
$$

This leads to the following expression for the partial gradient of the Lagrangian function with respect to $\mathbf{x}$ :

$$
\nabla_{\mathbf{x}} \mathcal{L}(\mathbf{x}, \boldsymbol{\lambda})=-2 \mathbf{H}^{T}(\mathbf{y}-\mathbf{H} \mathbf{x})+2 \boldsymbol{A} \mathbf{x} . \quad(\mathrm{B}-11)
$$

Equating this expression to zero gives the desired solution of $\mathbf{x}$ as

$$
\mathbf{x}(\boldsymbol{\lambda})=\left(\mathbf{H}^{T} \mathbf{H}+\boldsymbol{\Lambda}\right)^{-1} \mathbf{H}^{T} \mathbf{y} . \quad(\mathrm{B}-12)
$$

\section{SI Metric Conversion Factors}

$$
\begin{array}{rl}
c p \times 1.0^{*} & E-03=P a . s \\
f t \times 3.048^{*} & E-01=m \\
\text { md } \times 9.869223 & \text { E-04 }=\mu m^{2} \\
\text { psi } \times 6.894757 & \text { E+00 }=\mathrm{kPa}
\end{array}
$$

*Conversion factor is exact

SPEJ

Bülent Baygün is currently with Salomon Brothers Inc., working as an analyst in the Fixed Income Research Division, 7 World Trade Center, New York, New York 10048, email: bbaygun@zip.sbi.com. Prior to joining Salomon Brothers, he worked for Schlumberger-Doll Research, from 1992 to 1996 . He received BS and MS degrees in electrical engineering from the Middle East Technical University, Ankara, Turkey, in 1986 and 1988, respectively. He received an MS degree in mathematics in 1991 and a Ph.D. 
degree in electrical engineering in 1992, both from The University of Michigan.

Fikri J. Kuchuk is Chief Reservoir Engineer for Schlumberger Middle East, Schlumberger Technical Services, 8th Floor, Dubai International Trade Center, P. O. Box 9261, Dubai, United Arab Emirates, email: kuchuk@dubai.wireline.slb.com. He was a senior scientist and a program leader at Schlumberger-Doll Research Center, Ridgefield, Connecticut, USA. He was a consulting professor in the Petroleum Engineering Department of Stanford University from 1988 to 1994 . Before joining Schlumberger in 1982, he worked for Sohio Petroleum Company. He holds an MS degree from the Technical University of Istanbul, and MS and Ph.D. degrees from Stanford University, all in petroleum engineering.

Orhan Arikan is Assistant Professor of Electrical and Electronics Engineering at Bilkent University, Ankara, Turkey, email: oarikan@ee.bilkent.edu.tr. His current research interests are in adaptive signal processing, and time-frequency signal analysis. Before joining Bilkent University, he worked for three years as a Research Scientist at Schlumberger-Doll Research, Ridgefield, Connecticut, USA. He holds a B.Sc. Degree in Electrical Engineering from the Middle East Technical University and MS and Ph.D. degrees both in Electrical and Computer Engineering from the University of Illinois at UrbanaChampaign. 